\title{
THE PATH-PAIRABILITY NUMBER OF PRODUCT OF STARS
}

\author{
AdAm S. Jobson, André E. KÉzDy \\ University of Louisville, Louisville, KY 40292 \\ e-mail: adam.jobson@louisville.edu \\ andre.kezdy@louisville.edu \\ JENŐ LEHEL \\ University of Louisville, Louisville, KY 40292 \\ and \\ Alfréd Rényi Institute of Mathematics, Budapest, Hungary \\ e-mail: lyency@gmail.com \\ AND \\ GÁBOr MÉsZÁros \\ University of Memphis, Memphis, TN 38152 \\ e-mail: gmeszaros@memphis.edu
}

\begin{abstract}
The study of a graph theory model of certain telecommunications network problems lead to the concept of path-pairability, a variation of weak linkedness of graphs. A graph $G$ is $k$-path-pairable if for any set of $2 k$ distinct vertices, $s_{i}, t_{i}, 1 \leq i \leq k$, there exist pairwise edge-disjoint $s_{i}, t_{i}$-paths in $G$, for $1 \leq i \leq k$. The path-pairability number is the largest $k$ such that $G$ is $k$-path-pairable. Cliques, stars, the Cartesian product of two cliques (of order at least three) are 'fully pairable'; that is $\lfloor n / 2\rfloor$-pairable, where $n$ is the order of the graph. Here we determine the path-pairability number of the Cartesian product of two stars.
\end{abstract}

Keywords: path-pairability, weak linkage, Cartesian product, star-like network, telecommunications network.

2010 Mathematics Subject Classification: 05C38, 05C40. 
[1] L. Csaba, R.J. Faudree, A. Gyárfás, J. Lehel and R.H. Schelp, Networks communicating for each pairing of terminals, Networks 22 (1992) 615-626. doi:10.1002/net.3230220702

[2] R.J. Faudree, A. Gyárfás and J. Lehel, Three-regular path pairable graphs, Graphs Combin. 8 (1992) 45-52. doi:10.1007/BF01271707

[3] R.J. Faudree, A. Gyárfás and J. Lehel, Path-pairable graphs, J. Combin. Math. Combin. Comput. 29 (1999) 145-157.

[4] E. Győri, T.R. Mezei and G. Mészáros, Note on terminal-pairability in complete grid graphs, Discrete Math. 5 (2017) 988-990. doi:10.1016/j.disc.2017.01.014

[5] A. Huck, A sufficient condition for graphs to be weakly k-linked, Graphs Combin. 7 (1991) 323-351. doi:10.1007/BF01787639

[6] A.S. Jobson, A.E. Kézdy and J. Lehel, The path-pairability of the products of paths (2016), submitted.

[7] E. Kubicka, G. Kubicki, and J. Lehel, Path-pairable property for complete grids, in: Combinatorics, Graph Theory and Algorithms II (1999) 577-586.

[8] G. Mészáros, Linkedness and Path-Pairability in the Cartesian Product of Graphs, PhD Thesis (CEU, Budapest, 2015).

[9] G. Mészáros, On path-pairability in the Cartesian product of graphs, Discuss. Math. Graph Theory 36 (2016) 743-758.

doi:10.7151/dmgt.1888

Received 12 December 2016

Revised 27 December 2017

Accepted 27 December 2017 